\title{
Mouse Model of Heterotaxy with Single Ventricle Spectrum of Cardiac Anomalies
}

\author{
CHRISTINE N. AUNE, BISHWANATH CHATTERJEE, XIAO-QING ZHAO, RICHARD FRANCIS, LUCIANN BRACERO, \\ QING YU, JULIE ROSENTHAL, LINDA LEATHERBURY, AND CECILIA W. LO
}

\author{
Laboratory of Developmental Biology [C.N.A., B.C., X.-Q.Z., R.F., L.B., Q.Y., J.R., L.L., C.W.L.], National Heart, Lung, and Blood \\ Institute, National Institutes of Health, Bethesda, MD 20892; Department of Pediatrics [C.N.A.], Uniformed Services University, Bethesda, \\ MD 20814; Department of Pediatrics [C.N.A.], Walter Reed Army Medical Center, Washington, DC 20307
}

\begin{abstract}
Heterotaxy arises from a failure of the embryo to establish normal left-right asymmetry and is known to affect $3 \%$ of infants with congenital heart disease. A recessive mutation causing heterotaxy was recovered in a mouse mutagenesis screen focused on congenital heart defects. Homozygote mutants exhibit abnormal situs in the thoracic and abdominal cavities. Dextrocardia, levocardia, or mesocardia was seen together with right pulmonary isomerism and complex structural heart defects in the single ventricle spectrum. A dominant chamber of left ventricular morphology positioned on the left or right is seen together with transposition of the great arteries. Right atrial isomerism with or without total anomalous pulmonary venous connection was observed in half of the mutants. Because ciliary motion at the embryonic node is required for the specification of laterality, we examined the tracheal epithelia of newborn mice as a proxy for the nodal cilia. However, videomicroscopy showed no defect in ciliary motion. Genome scanning using polymorphic microsatellite markers mapped the mutation to a $3.3 \mathrm{Mb}$ interval on mouse chromosome 7. None of the genes previously described for familial heterotaxy were found in this interval, indicating a novel mutation in this mouse model of heterotaxy. (Pediatr Res 63: 9-14, 2008)
\end{abstract}

$\mathrm{T}$ he specification of laterality is essential for survival of air breathing mammals because left-right asymmetry associated with the four chamber heart and great vessels is required for the normal separation of pulmonary and systemic circulation. Heterotaxy syndromes involve birth defects in which organ situs in the thoracic and abdominal cavity are altered in a discordant manner. They are associated with $3 \%$ of all congenital heart defects (CHD) (1), with the prevalence estimated at 1.4/10,000 births (2). Because a wide spectrum of complex structural heart malformations are frequently seen in conjunction with heterotaxy, the overall prognosis is often poor. Some of the cardiovascular malformations associated

Received May 24, 2007; accepted August 14, 2007.

Correspondence: Cecilia W. Lo, Ph.D., NHLBI/NIH, 10 Center Drive MSC 1583, Bethesda, MD 20892-1583; e-mail: loc@nhlbi.nih.gov

This work was supported by funding from NHLBI DIR (ZO1-HL005701).

C.N.A., B.C., and X.-Q.Z. contributed equally to this work.

The views expressed in this article are those of the authors and do not reflect the official policy or position of the Department of the Army, Department of Defense, or the U.S. government. with heterotaxy syndromes include transposition of the great arteries, single ventricle defects, anomalous pulmonary venous connection, atrial septal defects, ventricular septal defects, and right ventricular outflow tract obstruction (3). Other associated findings include left-sided or bilobed liver, rightsided stomach with gut malrotation, and a high incidence of midline associated defects such as cleft lip/palate, encephalocele, and renal abnormalities $(1,3,4)$.

Despite detailed knowledge of the clinical presentations, the cause(s) of heterotaxy remains largely unknown. Data collected from the Baltimore-Washington Infant Study from 1980 to 1989 showed a potential association with a familial history of malformations (odds ratio $=5.1,95 \%$ confidence interval $=2.0-12.9$ ), maternal diabetes, and geographic location, suggesting both environmental and genetic contributions to heterotaxy syndromes (5). In addition, it has been reported that approximately $10 \%$ of infants with heterotaxy have a family history of a close relative with CHD (1). Several reports of familial cases have been described, showing transmission that is X-linked and autosomal recessive or dominant $(6-9)$. These studies identified mutations in three genes in the transforming growth factor- $\beta$ (TGF- $\beta$ ) signaling pathway (10). In addition, mutations have been found in CRELD1 (a cell adhesion molecule), the transcription factor NKX2.5, and zinc finger transcription factor ZIC3 (1). The latter was reported in four X-linked familial cases (2). Even though mutations have been identified in a few familial cases of heterotaxy, the genetic cause for the vast majority of familial and sporadic cases of heterotaxy remains unknown. Understanding the genetic contribution to heterotaxy is of importance not only for elucidating the underlying disease mechanism, but such genetic insights will also assist physicians in counseling families affected by heterotaxy syndromes.

Studies in animal models such as chick, Xenopus, zebrafish, and mice have generated a great deal of insight into the genes and cell signaling pathways that regulate left-right patterning

\footnotetext{
Abbreviations: CHD, congenital heart defect; EFIC, episcopic fluorescence image capture; PCD, primary ciliary dyskinesia; TAPVC, total anomalous pulmonary venous connection
} 
(10-12). Mice with left-right asymmetric four chamber hearts and separate pulmonary and systemic circulation are an ideal model organism for studying the complex structural heart defects that accompany abnormal left-right specification. The availability of knockout, knocking, transgenic, and gene trap methods for gene manipulations in mice also have greatly facilitated the genetic analysis of cell signaling pathways important in left-right patterning. One such pathway identified is sonic hedgehog $(S h h)$. Knockout mice deficient in Shh demonstrate CHDs with pulmonary isomerism and other features of heterotaxy (13). In addition, mutations affecting ciliary assembly or function have been shown to affect laterality. This reflects the essential role of the monocilia at the embryonic node in left-right specification (14). Interestingly, recent studies show Shh signaling is transduced by the ciliary axoneme $(15,16)$.

We recently recovered several mouse mutants exhibiting defects in laterality in a mouse mutagenesis screen for mutations causing CHD $(17,18)$. In this report, we present findings from one such mutant found to have abnormal situs in the thoracic and abdominal cavities together with preaxial polydactyly. Detailed analysis by histopathology showed these mutations phenocopy human heterotaxy syndromes. Analysis of ciliary function using the tracheal respiratory mucosa showed normal ciliary motion. This mutation was mapped to mouse chromosome 7 in a $3.3 \mathrm{Mb}$ interval with no genes known to be associated with heterotaxy or the specification of laterality. This mutant mouse model will be invaluable for studying the coordinate specification of organ situs and its dysregulation in heterotaxy syndromes.

\section{METHODS}

Breeding. Mice carrying the mutation of interest were originally obtained in the C57BL/6J (B6) background and were subsequently intercrossed with $\mathrm{C} 3 \mathrm{H} / \mathrm{HeJ}(\mathrm{C} 3 \mathrm{H})$ mice to generate $\mathrm{B} 6 / \mathrm{C} 3 \mathrm{H}$ hybrid offspring. These hybrid offspring were then intercrossed to recover homozygous fetuses with heterotaxy. Mutant animals were either obtained as newborn pups or were harvested from embryonic day 15 to 18.5 (E15.5 to E18.5). Each fetus or newborn pup was examined by necropsy followed by episcopic fluorescence image capture (EFIC) histology. In some cases, the trachea was harvested from newborn pups for analysis of ciliary motion in the tracheal mucosa by videomicroscopy. This animal study protocol has been approved by the National Heart, Lung, and Blood Institute Animal Care and Use Committee.
Necropsy and EFIC histology. Fetuses or neonatal stillborn pups were fixed in $10 \%$ buffered formalin. Necropsy was performed to examine the heart, great vessels, aortic arch sidedness, cavae, as well as ascertain organ situs in the thoracic and abdominal cavities. Some were further processed for imaging by EFIC (19). This entailed embedding the specimen in paraffin wax containing aniline dye, followed by sectioning using a Leica SM2500 microtome equipped with a Leica MZFLIII epifluorescence stereomicroscope (Wetzlar, Germany) and an Orca-ER digital camera (Hamamatsu, Hamamatsu City, Japan). Fluorescent images of the block face were captured serially to generate two-dimensional (2D) image stacks that are in perfect registration (19). For three-dimensional (3D) volume rendering, the 2D image stacks were processed using Improvision Volocity software (Coventry, UK). To refine diagnosis of complex structural heart defects, we also digitally resectioned the 2D image stacks in different imaging planes (19).

Videomicroscopy of ciliary motility. Trachea from newborn mice were removed, and tracheal rings were excised in L15 medium with $10 \%$ fetal bovine serum and placed on a upright Leica DMLFSA microscope and viewed with a $\times 63$ immersion lens at room temperature. The tracheal rings were positioned to optimize viewing of the motile cilia of the airway epithelium, and digital movies were captured using a Phantom v4.2 camera (Vision Research, Wayne, NJ) set at a frame rate of $800 \mathrm{~Hz}$. Homozygote mutant mice exhibiting preaxial duplication of the first digits and control littermates with normal digit patterning were examined in a similar manner.

Genome scan and linkage analysis. DNA was prepared from a small piece of the tail tissue and then polymerase chain reaction (PCR) amplified using dye-labeled primers for $48 \mathrm{~B} 6 / \mathrm{C} 3 \mathrm{H}$ polymorphic microsatellite markers. The resultant PCR products were pooled and separated by capillary electrophoresis on the Avant 3100 Genetic Analyzer (Applied Biosystems, Foster City, CA). The data generated were analyzed using the method of Neuhaus and Beier (20) for recombinant interval haplotype analysis. Thus, DNA markers located near the ends of each mouse chromosome were used to demarcate intervals that were treated as haplotypes for the purpose of linkage analysis (17). The frequency with which recombinant haplotypes are found across the entire genome in the fetuses with heterotaxy was used to statistically evaluate the chromosome location of the mutation.

\section{RESULTS}

Abnormal specification of abdominal and thoracic organ situs. A mutant was recovered from our mutagenesis screen exhibiting homozygous lethality associated with laterality defects and preaxial duplication on all four limbs (Fig. $1 E$ and $F)$. Situs abnormalities were associated with organs in both the thoracic and abdominal cavities. Mutants exhibited abnormal lung lobation consisting of four lung lobes symmetrically placed on both sides of the chest cavity (Fig. $1 A$ and $B$ ). This pattern, which normally is associated with the anatomic right, is indicative of right pulmonary isomerism. In addition, some animals exhibited abnormal heart situs and complex structural heart defects (Fig. 2). In contrast with the lung and heart, the
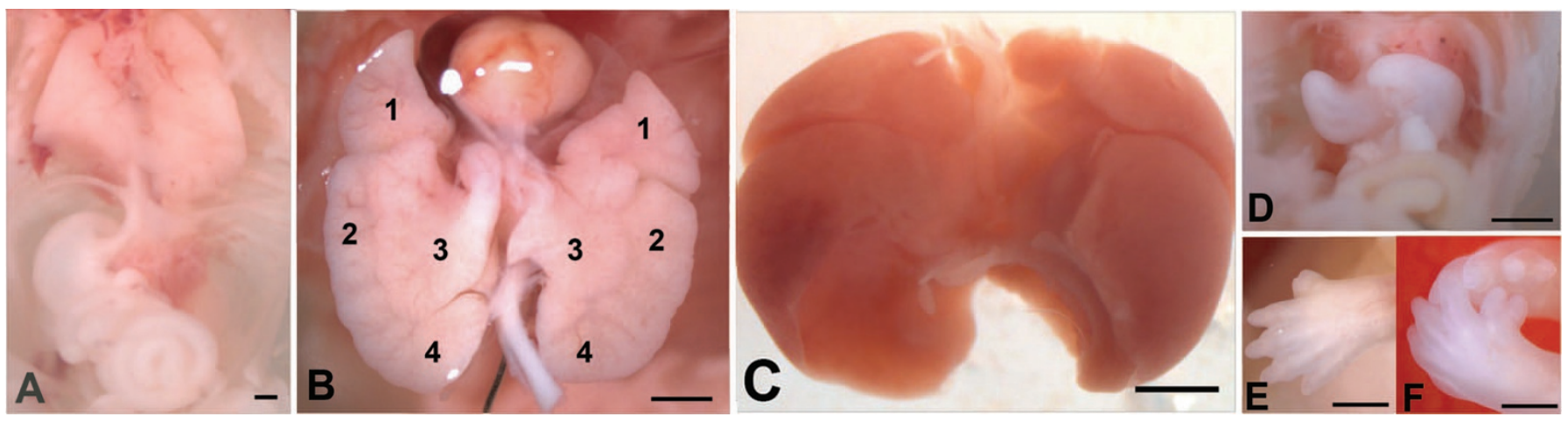

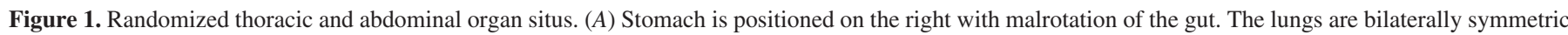

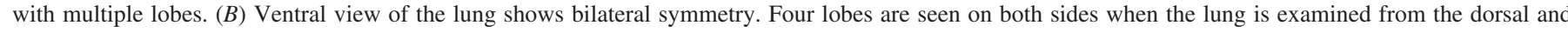

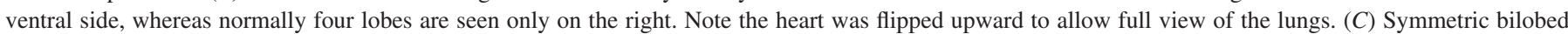

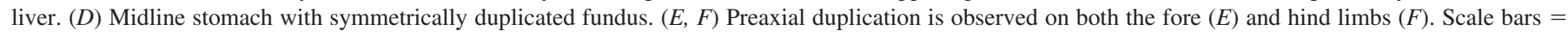
$1 \mathrm{~mm}$. 

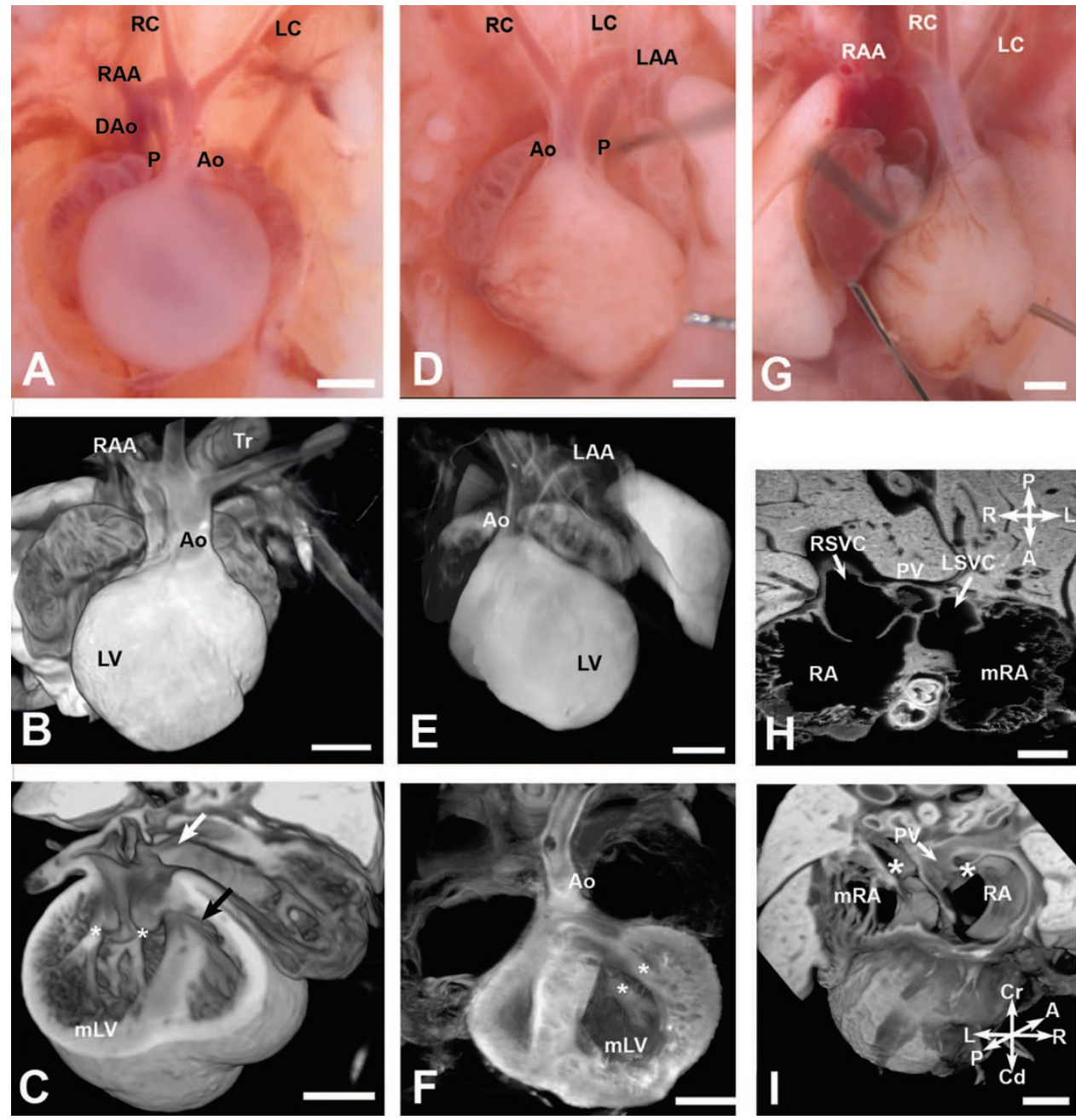

Figure 2. Heart situs anomalies with single ventricle spectrum of complex structural heart defects. $(A, B, C)$ Mesocardia is seen with anterior positioning of the aorta (Ao) and a right-sided aortic arch (RAA). EFIC reconstructed 3D images show the dominant chamber is a morphologic left ventricle (mLV), as indicated by attachment of the papillary muscles (asterisk in $C$ ) to the free wall. Note atrial and ventricular septal defects (white and black arrows, respectively), resulting in a common atrioventricular canal. P, pulmonary outflow; RC, right carotid; LC, left carotid; Tr: trachea. Scale bars $(A, B)=500$ um and $(C)=400$ um. $(D, E, F)$ Levocardia is seen with anterior positioning of the aorta and a left-sided aortic arch (LAA). EFIC reconstructions $(E, F)$ show a single dominant ventricle of left ventricular morphology $(\mathrm{mLV})$. Note papillary muscles attached to the free wall (asterisks in $F$ ). Scale bars $(D, E)=500$ um and $(F)=400$ um. $(G)$ Dextrocardia is seen with an anterior aorta and right-sided aortic arch (RAA). Scale bar $=500 \mathrm{um} .(H)$ Typically, mutants show right atrial isomerism, as indicated by insertion of the left and right superior vena cava (RSVC, LSVC) symmetrically into the anatomic right (RA) and left atria (mRA). This suggests the anatomic left atria is morphologically a right atria (mRA). Total anomalous pulmonary venous connection is indicated by insertion of the pulmonary vein (PV) confluence into the atrium on the right (RA). This image shows the aorta anterior and rightward of the pulmonary artery. Compass denotes orientation of section. A, anterior; P, posterior; L, left; R, right. Scale bar $=400$ um. $(I)$ Another section of the same heart shown in $H$ was 3D reconstructed and shown in a posterior view. Total anomalous pulmonary venous connection is indicated by insertion of the pulmonary vein (PV) confluence into the anatomic right atria (RA) near the midline (white arrow). The RSVC and LSVC (asterisks) are inserted symmetrically into the atria on the right and left, making the left-sided atrium a morphologic right atrium (mRA). There was no coronary sinus present. Compass denotes orientation of the 3D reconstruction. Cr, cranial; Cd, caudal; L, left; R, right; A, anterior; P, posterior. Scale bar $=400 \mathrm{um}$.

stomach was found either on the right or left side of the body (Fig. 1A; data not shown), and we also observed symmetrical bilobed liver (Fig. 1C). Together, these findings of abnormal organ situs in the thoracic and abdominal cavities indicated heterotaxy.

To assess the incidence of the various situs anomalies and the precise structural heart defects in this mutant mouse line, 10 homozygote mutant animals were analyzed by necropsy followed by serial section histology. We observed variation in heart situs, with three exhibiting dextrocardia, four with mesocardia, and three with levocardia. This was accompanied by either right- or left-sided aortic arch (Figs. $2 A, D$, and $G$ and 3). In contrast with the varied heart situs, the lungs of nine mutants showed right pulmonary isomerism. In the abdominal cavity, the stomach was right sided in four and left sided in five animals (Fig. 1A). One animal showed an unusual midline stomach with bilateral symmetry (Fig. 1D). Other abnormalities seen included hypoplastic kidney with abnormal ureter presentation, as well as asplenia or polysplenia (Fig. 3). Together, these findings are consistent with clinical features associated with heterotaxy syndromes.

Complex structural heart defects. The complex structural heart defects were analyzed using serial section histology carried out by EFIC imaging followed by 3D reconstructions (Fig. 2; also data not shown). This analysis showed that all mutants have a dominant ventricle of left ventricular morphol- 


\begin{tabular}{|c|c|c|c|c|c|c|c|c|c|}
\hline Stomach & Lung & Arch & Heart & $\begin{array}{c}\text { Left } \\
\text { Ventricle }\end{array}$ & Atria & AVC & $\begin{array}{c}\text { Anterior } \\
\text { Aorta }\end{array}$ & TAPVC & Spleen \\
\hline Right & RPI & RAA & Mesocardia & Right & $\begin{array}{c}\text { Right } \\
\text { Isomerism }\end{array}$ & Yes & Yes & No & Asplenia \\
\hline Left & RPI & RAA & Mesocardia & Right & $\begin{array}{c}\text { Right } \\
\text { Isomerism }\end{array}$ & Yes & Yes & Yes & Asplenia \\
\hline Left & RPI & LAA & Mesocardia & Left & $\begin{array}{c}\text { Right } \\
\text { Isomerism }\end{array}$ & Yes & Yes & Yes & Asplenia \\
\hline Left & Normal & LAA & Mesocardia & Left & Normal & Yes & Yes & No & Asplenia \\
\hline Bilateral & RPI & RAA & Dextrocardia & Right & $\begin{array}{c}\text { Right } \\
\text { Isomerism }\end{array}$ & Yes & Yes & Yes & Poly \\
\hline Right & RPI & LAA & Levocardia & Left & Normal & Yes & Yes & No & Asplenia \\
\hline Left & RPI & RAA & Dextrocardia & Right & $\begin{array}{c}\text { Right } \\
\text { Isomerism }\end{array}$ & Yes & Yes & Yes & Asplenia \\
\hline Left & RPI & LAA & Levocardia & Left & Normal & Yes & Yes & No & Asplenia \\
\hline Right & RPI & LAA & Levocardia & Right & Normal & Yes & Yes & Yes & Poly \\
\hline Right & RPI & LAA & Dextrocardia & Left & $\begin{array}{c}\text { Right } \\
\text { Isomerism }\end{array}$ & Yes & Yes & No & Asplenia \\
\hline
\end{tabular}

\section{Inverted Situs}

Midline Symmetric

\section{Right Sided Isomerism}

Figure 3. Summary of cardiovascular and situs anomalies. The situs anomalies and complex structural heart defects in 10 mutant embryos were analyzed by necropsy followed by EFIC histology and EFIC 3D reconstructions. Situs of the stomach and heart were randomized and showed no coordination. However, heart situs were largely aligned with the aortic arch, with dextrocardia usually linked with right-sided aortic arch (RAA). Almost all embryos showed right pulmonary isomerism (RPI), anterior positioning of the aorta, polysplenia (Poly), and total anomalous pulmonary venous connection (TAPVC). LAA, left-sided aortic arch; AVC, atrioventricular canal defect.

ogy (Fig. 2). Interestingly, the dominant chamber exhibited varied situs, with five positioned on the anatomic right and five on the anatomic left (Figs. $2 A-F$ and 3). Those positioned on the anatomic right can be considered as inverted ventricles. We note the small chamber did not show the trabeculation typical for the right ventricle. Nor did it have the trabecula septomarginalis, a band of muscle situated at the roof of the right ventricle that normally subdivides the inlet versus outlet portion of the right ventricular chamber (21). Together, these findings suggest this mutant has a single ventricle spectrum of heart defect that is characterized by a dominant chamber of left ventricular morphology.

All mutants exhibited malalignment of the great arteries with anterior positioning of the aorta or transposition of the great arteries (Figs. 2 and 3). In two animals, a functional single ventricle is indicated by the insertion of both outflows into the small chamber. All mutants also had atrial and ventricular septal defects that resulted in atrioventricular canals (Fig. 2C). Half of the mutants exhibited right atrial isomerism (Fig. 3). This is indicated by the insertion of the left and right superior vena cava (LSVC and RSVC) into the anatomic left and right atria with no coronary sinus (Fig. $2 H$ ). This contrasts with normal insertion of the RSVC into the right atria and the LSVC into the coronary sinus, which then enters the right atria. Six of the 10 mutants also showed total anomalous pulmonary venous connection (TAPVC) (Fig. 3).
An example of this is shown in Fig. $2 H$ and $I$, where the pulmonary vein confluence can be seen to connect with the right-sided atrium near the midline.

Overall, these findings show a consistent phenotype comprised of a dominant chamber of left ventricular morphology and endocardial cushion defects together with right pulmonary isomerism and transposed positioning of the great arteries. All six hearts with right atrial isomerism also exhibited right pulmonary isomerism. Beyond this, the overall patterning of the aortic arch, atria, and heart and stomach situs were varied, with no correlation between situs in the thoracic and abdominal cavities. However, it is interesting to note that four of five animals with TAPVC and all animals with right-sided aortic arch exhibited inverted ventricles.

Analysis of ciliary motility by videomicroscopy. The specification of laterality is known to involve ciliary motility at the embryonic node. In primary ciliary dyskinesia (PCD) and Kartagener's syndrome, patients exhibit situs inversus accompanied by sinopulmonary disease caused by immotile or dyskinetic ciliary motion associated with the airway mucosa $(22,23)$. Similar findings have been reported in studies of a mouse model of PCD $(24,25)$. To ascertain whether situs anomalies in these mutants may involve defects in ciliary motility, we examined ciliary function in the respiratory mucosa of newborn mice as a proxy for the nodal cilia. Tracheal mucosae harvested from liveborn homozygote mutants were 


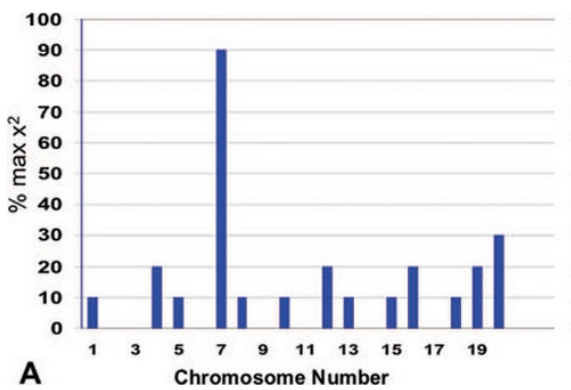

\begin{tabular}{|l|l|l|l|l|l|l|}
\hline Mutant ID & & Mutant 1 & Mutant 2 & Mutant 3 & Mutant 4 & Mutant 5 \\
\hline Marker ID & MB & & & & & \\
\hline D7mit152 & 4.3 & & & & & \\
\hline D7mit56 & 16.1 & & & & & \\
\hline D7mit191 & 18.1 & & & & & \\
\hline D7mit192 & 24.7 & & & & & \\
\hline D7mit266 & 28 & & & & & \\
\hline D7mit228 & 39.7 & & & & & \\
\hline D7mit350 & 83.4 & & & & & \\
\hline D7mit229 & 125 & & & & & \\
\hline
\end{tabular}

B

Figure 4. Genome scan analysis and fine mapping of the mutation. (A) Mutation was mapped to chromosome 7 using interval haplotype analysis. A max $\mathrm{x}^{2}$ of greater than 75\% indicates the mutation is located on mouse chromosome 7 with $p>95 \%$. (B) Mutation was generated in a B6 (pink) strain background, and, with subsequent outcross into a $\mathrm{C} 3 \mathrm{H}$ (blue) background, the mutation could be mapped by examining regions of B6 homozygosity linked to the mutant phenotype. This localized the mutation to microsatellite markers D7mit192 and D7mit266, a 3.3 Mb interval on mouse chromosome 7. Yellow denotes markers that were B6/C3H heterozygous.

examined by videomicroscopy. In normal littermates comprised of wild-type and heterozygous mutants, very rapid and coordinated ciliary beat was observed. This same rapid ciliary beat was also observed in the tracheal mucosa of homozygote mutant mice. These findings suggest ciliary function is not altered in this mutant mouse model of heterotaxy.

Mutation mapped to mouse chromosome 7. To map the mutation, heterozygote mutants in the B6 background were crossed to $\mathrm{C} 3 \mathrm{H}$ mice to generate $\mathrm{B} 6 / \mathrm{C} 3 \mathrm{H}$ hybrid offspring. These hybrid offspring were intercrossed, and fetuses were harvested between E15.5 and birth. DNA obtained from 15 mutant fetuses exhibiting heterotaxy was analyzed by genome scanning using 48 microsatellite DNA markers polymorphic between $\mathrm{B} 6$ and $\mathrm{C} 3 \mathrm{H}$ inbred mouse strains. These DNA markers, positioned near the ends of each mouse chromosome, were used to demarcate intervals that are treated as haplotypes for the purpose of linkage analysis (20). For the larger mouse chromosomes (chromosomes 1, 2, 3, 4, and 6), one or two additional markers were included. The mutation is expected to lie in a chromosome interval that is consistently B6 homozygous. Analysis of the genome scanning data localized the mutation to mouse chromosome 7 (Fig. 4A). To refine the map interval on chromosome 7, another 130 mutants were analyzed using an additional 18 microsatellite markers and 16 single nucleotide polymorphic markers. This analysis narrowed the interval to a $3.3 \mathrm{Mb}$ region positioned between markers D7mit192 and D7mit266 (Fig. 4B). Search of the mouse genome database showed no genes known to be linked with heterotaxy within this chromosomal interval.

\section{DISCUSSION}

We recovered a recessive mutation causing a phenotype consistent with features of human heterotaxy syndromes. Homozygote mutants exhibit abnormal organ situs in both the thoracic and abdominal cavities, with most showing right pulmonary isomerism and anterior placement of the aorta. All mutants exhibit atrioventricular canal defect with a single ventricle phenotype consisting of a dominant ventricle of left-sided morphology, with half positioned on the left and half on the right side of the chest cavity. TAPVC and right atrial isomerism were also seen in half of the animals. Given recent findings indicating an essential role for the cilia in breaking of symmetry in the early embryo (26-30), we examined ciliary function in the respiratory mucosal epithelia as a proxy for the nodal cilia. No detectable defect was seen in ciliary motion, indicating that this mutation causing heterotaxy is not likely to involve defects in nodal ciliary motion. However, it is interesting to note that a recent study showed an association of heterotaxy with PCD (31).

We mapped the mutation causing heterotaxy to a $3.3 \mathrm{Mb}$ interval of mouse chromosome 7 , a region containing a total of 72 genes. None of the genes known to be associated with human heterotaxy syndromes are localized in this region (22). However, we note the presence of $T G F-\beta 1$, which is known to play an important role in left-right specification. Laterality defects are seen in knockout mice deficient in activin receptor IIB (Acvr $2 b)$, a receptor that binds and transduces TGF- $\beta$ signals (32), and mutations in Acvr2b have been observed in heterotaxy patients $(1,2)$. The mapped interval includes another gene of interest, Numb-L, a regulator of Notch signaling (33-35). Over-expression of znumb in zebrafish embryos has been shown to cause laterality defects associated with the heart tube (36). However, sequencing of the coding regions of $T G F-\beta 1$ and $N u m b-L$ failed to show any sequence changes. A bacterial artificial chromosome library is currently being constructed from the genomic DNA from this heterotaxy mutant to facilitate recovery of the mutation. Identification of this novel mutation may provide further insights into the genetic origin of human heterotaxy syndromes.

Clinically, the combination of heterotaxy with complex $\mathrm{CHD}$ has a poor prognosis, even with optimal medical management (37). Thus, patients with CHD requiring surgical management as a functional single ventricle must undergo three operations and suffer from some of the highest mortality rates $(38,39)$. Insights gained from identifying the underlying genetic defects of heterotaxy will generate hypotheses about developmental mechanisms causing these complex heart defects. Knowledge gained from such studies may improve parental counseling and the future development of diagnostic and therapeutic strategies for heterotaxy syndromes.

\section{REFERENCES}

1. Belmont JW, Mohapatra B, Towbin JA, Ware SM 2004 Molecular genetics of heterotaxy syndromes. Curr Opin Cardiol 19:216-220 
2. Ware SM, Peng J, Zhu L, Fernbach S, Colicos S, Casey B, Towbin J, Belmont JW 2004 Identification and functional analysis of ZIC3 mutations in heterotaxy and related congenital heart defects. Am J Hum Genet 74:93-105

3. Bartram U, Wirbelauer J, Speer CP 2005 Heterotaxy syndrome: asplenia and polysplenia as indicators of visceral malposition and complex congenital heart disease. Biol Neonate 88:278-290

4. Ticho BS, Goldstein AM, Van Praagh R 2000 Extracardiac anomalies in the heterotaxy syndromes with focus on anomalies of midline-associated structures. Am J Cardiol 85:729-734

5. Kuehl KS, Loffredo C 2002 Risk factors for heart disease associated with abnormal sidedness. Teratology 66:242-248

6. Alonso S, Pierpont ME, Radtke W, Martinez J, Chen SC, Grant JW, Dahnert I, Taviaux S, Romey MC, Demaille J, Bouvagnet P 1995 Heterotaxia syndrome and autosomal dominant inheritance. Am J Med Genet 56:12-15

7. Casey B, Cuneo BF, Vitali C, van Hecke H, Barrish J, Hicks J, Ballabio A, Hoo JJ 1996 Autosomal dominant transmission of familial laterality defects. Am J Med Genet 61:325-328

8. Arnold GL, Bixler D, Girod D 1983 Probable autosomal recessive inheritance of polysplenia, situs inversus and cardiac defects in an Amish family. Am J Med Genet $16: 35-42$

9. Kosaki K, Casey B 1998 Genetics of human left-right axis malformations. Semin Cell Dev Biol 9:89-99

10. Mercola M, Levin M 2001 Left-right asymmetry determination in vertebrates. Annu Rev Cell Dev Biol 17:779-805

11. Yost HJ 2001 Establishment of left-right asymmetry. Int Rev Cytol 203:357-381

12. Shiratori H, Hamada H 2006 The left right axis in mouse: from origin to morphology. Development 133:2095-2104

13. Tsukui T, Capdevila J, Tamura K, Ruiz-Lozano P, Rodriguez-Esteban C, YoneiTamura S, Magallon J, Chandraratna RA, Chien K, Blumberg B, Evans RM, Belmonte JC 1999 Multiple left-right asymmetry defects in Shh(-/-) mutant mice unveil a convergence of the shh and retinoic acid pathways in the control of Lefty-1. Proc Natl Acad Sci U S A 96:11376-11381

14. Okada Y, Takeda S, Tanaka Y, Belmonte JC, Hirokawa N 2005 Mechanism of noda flow: a conserved symmetry breaking event in left-right axis determination. Cell 121:633-644

15. Caspary T, Larkins CE, Anderson KV 2007 The graded response to sonic hedgehog depends on cilia architecture. Dev Cell 12:767-778

16. Liu A, Wang B, Niswander LA 2005 Mouse intraflagellar transport proteins regulate both the activator and repressor functions of Gli transcription factors. Development 132:3103-3111

17. Yu Q, Shen Y, Chatterjee B, Siegfried BH, Leatherbury L, Rosenthal J, Lucas JF, Wessels A, Spurney CF, Wu YJ, Kirby ML, Svenson K, Lo CW 2004 ENU induced mutations causing congenital cardiovascular anomalies. Development 131:62116223

18. Shen Y, Leatherbury L, Rosenthal J, Yu Q, Pappas MA, Wessels A, Lucas J, Siegfried B, Chatterjee B, Svenson K, Lo CW 2005 Cardiovascular phenotyping of fetal mice by noninvasive high-frequency ultrasound facilitates recovery of ENU induced mutations causing congenital cardiac and extracardiac defects. Physiol Genomics 24:23-36

19. Rosenthal J, Mangal V, Walker D, Bennett M, Mohun TJ, Lo CW 2004 Rapid high resolution three dimensional reconstruction of embryos with episcopic fluorescence image capture. Birth Defects Res C Embryo Today 72:213-223

20. Neuhaus IM, Beier DR 1998 Efficient localization of mutations by interval haplotype analysis. Mamm Genome 9:150-154
21. Wenink AC 1981 Embryology of the ventricular septum. Separate origins of its components. Virchows Arch A Pathol Anat Histol 390:71-79

22. van's Gravesande KS, Omran H 2005 Primary ciliary dyskinesia: clinical presentation, diagnosis and genetics. Ann Med 37:439-449

23. De Iongh RU, Rutland J 1995 Ciliary defects in healthy subjects, bronchiectasis, and primary ciliary dyskinesia. Am J Respir Crit Care Med 151:1559-1567

24. Fliegauf M, Olbrich H, Horvath J, Wildhaber J, Zariwala M, Kennedy M, Knowles MR, Omran H 2005 Mislocalization of DNAH5 and DNAH9 in respiratory cells from patients with primary ciliary dyskinesia. Am J Respir Crit Care Med 171:13431349

25. Ibanez-Tallon I, Gorokhova S, Heintz N 2002 Loss of function of axonemal dynein Mdnah5 causes primary ciliary dyskinesia and hydrocephalus. Hum Mol Genet 11:715-721

26. Okada Y, Nonaka S, Tanaka Y, Saijoh Y, Hamada H, Hirokawa N 1999 Abnormal nodal flow precedes situs inversus in iv and inv mice. Mol Cell 4:459-468

27. Peeters H, Devriendt K 2006 Human laterality disorders. Eur J Med Genet 49:349_ 362

28. Satir P, Christensen ST 2007 Overview of structure and function of mammalian cilia Annu Rev Physiol 69:377-400

29. Nonaka S, Tanaka Y, Okada Y, Takeda S, Harada A, Kanai Y, Kido M, Hirokawa N 1998 Randomization of left-right asymmetry due to loss of nodal cilia generating leftward flow of extraembryonic fluid in mice lacking KIF3B motor protein. Cell 95:829-837

30. Marszalek JR, Ruiz-Lozano P, Roberts E, Chien KR, Goldstein LS 1999 Situs inversus and embryonic ciliary morphogenesis defects in mouse mutants lacking the KIF3A subunit of kinesin-II. Proc Natl Acad Sci USA 96:5043-5048

31. Kennedy MP, Omran H, Leigh MW, Dell S, Morgan L, Molina PL, Robinson BV Minnix SL, Olbrich H, Severin T, Ahrens P, Lange L, Morillas HN, Noone PG, Zariwala MA, Knowles MR 2007 Congenital heart disease and other heterotaxic defects in a large cohort of patients with primary ciliary dyskinesia. Circulation 115:2814-2821

32. Oh SP, Li E 1997 The signaling pathway mediated by the type IIB activin receptor controls axial patterning and lateral asymmetry in the mouse. Genes Dev 11:18121826

33. Qin H, Percival-Smith A, Li C, Jia CY, Gloor G, Li SS 2004 A novel transmembrane protein recruits numb to the plasma membrane during asymmetric cell division. J Biol Chem 279:11304-11312

34. Johnson JE 2003 Numb and Numblike control cell number during vertebrate neurogenesis. Trends Neurosci 26:395-396

35. Uemura T, Shepherd S, Ackerman L, Jan LY, Jan YN 1989 numb, a gene required in determination of cell fate during sensory organ formation in Drosophila embryos. Cell 58:349-360

36. Niikura Y, Tabata Y, Tajima A, Inoue I, Arai K, Watanabe S 2006 Zebrafish Numb homologue: phylogenetic evolution and involvement in regulation of left-right asymmetry. Mech Dev 123:407-414

37. Kim SJ, Kim WH, Lim HG, Lee CH, Lee JY 2006 Improving results of the Fontan procedure in patients with heterotaxy syndrome. Ann Thorac Surg 82:1245-1251

38. Cohen MS, Schultz AH, Tian ZY, Donaghue DD, Weinberg PM, Gaynor JW, Rychik J 2006 Heterotaxy syndrome with functional single ventricle: does prenata diagnosis improve survival? Ann Thorac Surg 82:1629-1636

39. Morales DL, Braud BE, Booth JH, Graves DE, Heinle JS, McKenzie ED, Fraser CD Jr 2006 Heterotaxy patients with total anomalous pulmonary venous return: improving surgical results. Ann Thorac Surg 82:1621-1627; discussion 1627-1628 